\title{
Who suffers from pharmaceutical poverty and what are their needs? Evidence from a Spanish region.
}

\section{Maria Rubio-Valera ( $\nabla$ mrubio@pssjd.org )}

https://orcid.org/0000-0003-2856-1697

\section{Silvia Marqués-Ercilla}

Institut Catala De La Salut

\section{Maria Teresa Peñarrubia-María}

Idiap Research Institute

\section{Rosa María Urbanos-Garrido}

Universidad Complutense de Madrid

\section{Carme Borrell}

Agencia de Salut Publica de Barcelona

Jordi Bosch

Banco Farmacéutico

Alba Sánchez-Viñas

Fundacio Sant Joan de Deu

Ignacio Aznar-Lou

PRISMA Research Group (Sant Joan de Déu Research Institute / CIBERESP) https://orcid.org/00000002-6780-5968

\section{Research}

Keywords: Healthcare Disparities, Health Services Accessibility, Pharmaceutical Poverty, Pharmaceutical Preparations

Posted Date: May 29th, 2020

DOI: https://doi.org/10.21203/rs.3.rs-31755/v1

License: (9) This work is licensed under a Creative Commons Attribution 4.0 International License. Read Full License 


\section{Abstract \\ Background}

Pharmaceutical poverty occurs when a patient cannot afford the cost of prescribed medication and/or medical products. Non-profit organisations are covering the cost of medication to those patients in some contexts. The aim of the study was to describe the population of beneficiaries of the PB, a nongovernmental organization which provides free of charge access to medicines, and their utilization pattern of medicines and healthcare products.

\section{Methods}

This was an observational study using PB beneficiary data collected between November 2017 and December 2018 in Catalonia. The Catalan Health Service provided information from the general population. A descriptive analysis of the beneficiaries' characteristics was conducted and compared to general population.

\section{Results}

The beneficiaries $(N=1206)$ were mainly adults with a low level of education, unemployed, with functional disability and with $\geq 1$ children. Compared with the general population, the beneficiaries were older, had a lower level of education, showed a higher prevalence of functional disability, were less likely to be Spanish, and more likely to be divorced and unemployed. The beneficiaries were polymedicated and most were using medication related to the nervous (79\%), musculo-skeletal (68\%) and cardiovascular system (56\%) and alimentary tract and metabolism (68\%). Almost $19 \%$ of beneficiaries used healthcare products. Female beneficiaries were older and more likely to be divorced or widowed, employed and with children. Compared to men, women were more likely to use medicines for pain and mental disorders. The paediatric group used medications for severe, chronic conditions (heart diseases, autoimmune diseases, conduct disorders and attention deficit hyperactivity disorder).

\section{Conclusions}

Patients with severe, chronic and disabling conditions are affected by pharmaceutical poverty. While the system of co-payment remains unchanged, physicians and paediatricians should explore economic barriers to treatment and direct their patients to resources that help to cover the cost of treatment.

\section{Introduction}

Universal healthcare coverage aims to ensure that everyone can use necessary health services without experiencing financial hardship. However, medicines are subject to out-of-pocket payments worldwide [1]. 
The cost of medicines has been systematically identified as a factor that limits adherence to medications, mainly for disadvantaged populations [2-4]. The failure to effectively use prescription medicines can lead to worse health status and higher costs to the healthcare system [5]. Non-adherence worsens health outcomes and increases the risk of mortality and hospitalization [6, 7]. Greater use of medical services and productivity losses are also consequences of from medication non-adherence, which places a significant cost burden on healthcare systems $[8,9]$. Non-adherence is higher when more expensive medicines are prescribed and when co-payment is applied $[4,10,11]$; interventions that reduce the co-payment contributions or exempt the patient from payment have effectively proven to reduce nonadherence $[12,13]$.

The World Health Organization (WHO) defines catastrophic health spending as those health expenditures "greater than or equal to $40 \%$ of a household's non-subsistence income, i.e., income available after basic needs have been met" [14]. According to a recent report by the WHO, medicines are the main cause of outof-pocket payments incurred by households with catastrophic health spending (i.e., households that cannot afford to meet basic needs like food, housing and heating because of a health-related condition) [1]. Catastrophic health spending may lead to or deepen poverty, undermine health, and exacerbate health and socioeconomic inequalities [1].

In Spain, the National Health System (NHS) is taxpayer-funded, covers residents and foreign nationals and, with a few exceptions, is free at point of delivery. In Catalonia, one of the most populated regions in Spain, where 7.6 million people live, there are 371 publicly financed primary care centres that manage most of the prescriptions [15]. Prescription and over-the-counter pharmacological treatments are dispensed in privately managed community pharmacies. Patients pay a part of the cost of prescription medication, with the share dependent on the medication and patients' characteristics. The co-payment applies to pensioners and non-pensioners and ranges from 0-60\% depending on annual income, with a $10 \%$ reduced contribution for some medications (most chronic treatments) [16]. Upper limits to copayments are set only for pensioners, ranging from around 8 to 62 euros per month, and all patients pay $100 \%$ of the cost of medicines not covered by the NHS. Over $70 \%$ of primary care attendees have an assigned co-payment of $40 \%$ or $10 \%$ [17]. Although some particular groups are exempt from co-payment (disabled patients, people receiving non-contributory pensions, unemployed people whose benefits expired or beneficiaries of social integration subsidies), in $2018,3 \%$ of Spaniards reported not having taken a medication prescribed in a public healthcare centre because they could not afford it [18].

In response to this situation, in recent years several non-governmental organizations (NGOs) have provided free of charge access to medicines. This is the case of the NGO Pharmaceutical Bank (PB) which, in 2015, launched a pilot program in Barcelona - the Social Medicine Fund (SMF)- to fight against what was called "pharmaceutical poverty". The NGO, in collaboration with the Catalan Health Institute (which manages $80 \%$ of Catalan primary care centres) and the local council, began to cover the cost of prescribed medication and medical products to those patients attended in primary care centres who could not afford their treatments. Since then, the program has been further extended and is now available not 
only in the city of Barcelona, but also in eight other cities in the metropolitan area and some cities outside the region of Catalonia.

In order to benefit from the SMF, patients must first visit primary care social workers who corroborate their medical needs and verify their financial situation, through inspection of tax returns, payrolls and rent or mortgage receipts, etc. Social workers provide the beneficiaries with a list of partner community pharmacies where they can get the medicines free-of-charge. The beneficiaries have to fulfil the following criteria: 1 ) to be attended in a Catalan Health Institute primary care centre; 2 ) to require a treatment for at least 6 months that has a monthly cost of 20 Euros or more; and 3) to be in a situation of financial hardship (defined as a monthly household income after discounting rent or mortgage, lower than €550 for one person, $€ 670$ for two people or $€ 670$ plus $€ 75$ per additional person).

The SMF covers the patients' total treatment cost for a period of six months, which may be renewed if the conditions have not changed. The aid covers the cost of prescription medicines and healthcare products. In exceptional cases, when it is clinically relevant, the SMF also covers the cost of over-the-counter medicines. The NGO Pharmaceutical Bank is partnered with Catalan Health Institute primary care professionals and pharmacists from several community pharmacies. In Catalonia in 2018, 124 primary care centres and 407 community pharmacies were collaborating with the SMF.

There is some evidence on inadequate access to medicines in developing countries $[19,20]$ but, to the best of our knowledge, no previous study has provided information on the use of medicines by patients in a situation of pharmaceutical poverty or on the characteristics of these patients in Western societies. This paper gives a detailed description of SMF beneficiaries and of their pattern of utilization of medicines and healthcare products, which is compared with the pattern of use in the general population in Catalonia.

\section{Methods}

\section{Design}

This was an observational study using data from the Pharmaceutical Bank NGO database, collected between November 2017 and December 2018 in Catalonia.

\section{Variables}

Our database includes information on beneficiaries' characteristics, including gender (female, male), age, educational level (without formal education, and primary, secondary, or higher education), nationality (Spanish; other), presence of functional disability and assigned percentage of co-payment $(10 \%, 40 \%$, $50 \%$ or $60 \%$ ). In the adult population ( $\geq 18$ years old), civil status (married, single, divorced, widowed, other), employment status (active, unemployed with benefits, pensioner or beneficiary of social assistance, unemployed without benefits) and number of children were also registered. 
Individuals were divided into 3 age groups ( $0-14$ years old, $15-64$ years old and $\geq 65$ years old). Number of children was also categorized $(0,1,2,3,4$ or more).

Permanence in the SMF was estimated by taking into account the number of renewals and classified as no renewal (6 months), one renewal (6 to12 months) or more.

Medicines provided by the NGO were classified using the Anatomical Therapeutic Chemical (ATC) system. The selected healthcare products were those most frequently used: wound dressings, surgical wound dressings, varicose vein stockings and socks, inhalation chambers, diapers, allergen-specific immunotherapy, with a final category left for other products.

The Catalan Health Survey 2018 (ESCA; Enquesta de Salut de Catalunya) was used as a source of data from the general Catalan population. ESCA is an official survey consisting of a multistage probability sample representative of non-institutionalized residents of Catalonia, which includes information about personal characteristics, lifestyles, use of health services and health indicators. Finally, The Catalan health Service provided information on the use of publicly financed medicines in 2018 for the total Catalan population.

\section{Analysis strategy}

A descriptive analysis of the beneficiaries' characteristics was conducted using means for continuous variables and proportions for categorical variables. Using data from the ESCA, the characteristics of the general Catalan population were also described. Probability weights were applied to ensure representativeness.

First, we calculated the proportion of beneficiaries that received at least one medication by anatomical group and therapeutic subgroup, and the proportion of beneficiaries that received at least one healthcare product. Then, the overall use of medicines and healthcare products covered by the NGO and CatSalut was described (frequency and proportion of the total number of medicines - anatomical groups and therapeutic subgroups - and healthcare products were estimated). The profiles of medicine and healthcare product use are shown by gender and age group $(0-14,15-64$ and $\geq 65$ years old).

\section{Results}

Characteristics of beneficiaries

Between November 2017 and December 2018, 1206 people benefited from the SMF and 227 (18.8\%) and $147(12.2 \%)$ renewed the help once and twice, respectively. Almost $96 \%$ (1154) of beneficiaries had an assigned co-payment rate of $40 \%$ (corresponding to non-pensioners with annual incomes up to $18,000 €$ ), $3.1 \%$ (37) had a co-payment rate of $10 \%$ and the rest had a co-payment rate of $50 \%$ or $60 \%$.

Table 1 compares the sociodemographic characteristics of beneficiaries of the NGO Pharmaceutical Bank in Catalonia and those of the general Catalan population. Mean age was almost 49 years old and 
the $52 \%$ of the beneficiaries were female. Most of the population was Spanish (62.1\%) and a third presented functional disability. Among the adult population, most had primary or no formal education (68.3\%); were unemployed without benefits (57.0\%); and had up to two children. Most common civil status in the adult population was married (38.4\%), followed by single (28.9\%), and divorced or separated (23.1\%). 
Table 1

Sociodemographic characteristics of the population

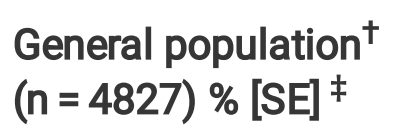

General population ${ }^{\dagger}$

$(n=4827) \%[S E]^{\ddagger}$
PB beneficiaries $(n=1206) \%$

(n) $\neq$
Female beneficiaries ( $\mathrm{n}$ $=623$ )
Male beneficiaries

$(n=583)$

Mean age (SE)

$42.3(0.31)$

$48.9(0.53)$

$51.0(0.70)$

$46.6(0.78)$

Age group

0-14 years old

$15.6[0.47]$

$8.6(104)$

6.7 (42)

$10.8(63)$

15-64 years old

$65.8[0.67]$

76.1 (918)

75.1 (468)

77.5 (452)

$\geq 65$ years old

18.7 [0.55]

15.3 (184)

18.1 (113)

11.7 (68)

Female

$50.6[0.75]$

51.7 (623)

Functional disability

11.8 [0.46]

34.7 (419)

$36.3(226)$

33.1 (193)

Nationality

Spanish

$85.7[0.54]$

62.1 (749)

64.0 (399)

$60.0(350)$

Other

11.8 [0.50]

37.5 (452)

$35.5(221)$

$39.6(231)$

Spanish and other

$2.5[0.24]$

Missing

ADULT POPULATION

( $\geq 18$ years old)

na

na

na

0.4 (5)

$0.5(3)$

$0.3(2)$

$(n=3600)$

$(n=1065)$

$(n=570)$

$(n=495)$

\section{Educational level}

Without education or

primary education

Secondary education

Higher education

$58.5[0.84]$

$25.5(272)$

24.1 (150)

$24.2(141)$

$21.6[0.72]$

$6.0(64)$

$5.3(33)$

$5.5(32)$

${ }^{+}$Data from the Catalan Health Survey (Enquesta de Salut de Catalunya (ESCA)) in 2018.

‡With the exception of Mean age (mean (SE)).

$\S$ Data from the Catalan Health Survey (ESCA) in 2014 (the last year this data was collected) $(n=$ 1804).

PB: Pharmaceutical Bank; na: Data not available or not applicable.

Bold numbers indicate statistically significant differences $(p<0.05)$ between male and female beneficiaries on Students' $T$ test for mean age and chi-squared tests for categorical variables. 


\begin{tabular}{|c|c|c|c|c|}
\hline & $\begin{array}{l}\text { General population }^{\dagger} \\
(n=4827) \%[S E]^{\ddagger}\end{array}$ & $\begin{array}{l}\text { PB } \\
\text { beneficiaries } \\
(n=1206) \% \\
(n)^{\ddagger}\end{array}$ & $\begin{array}{l}\text { Female } \\
\text { beneficiaries ( } \mathrm{n} \\
=623 \text { ) }\end{array}$ & $\begin{array}{l}\text { Male } \\
\text { beneficiaries } \\
(n=583)\end{array}$ \\
\hline Missing & $0.3[0.09]$ & $0.2(2)$ & $0.2(1)$ & $0.2(1)$ \\
\hline \multicolumn{5}{|l|}{ Civil status§ } \\
\hline Married & $57.2[1.25]$ & $38.4(409)$ & $33.2(189)$ & $44.4(220)$ \\
\hline Single & $28.8[1.14]$ & $28.9(308)$ & $25.4(145)$ & $32.9(163)$ \\
\hline Divorced or separated & $6.0[0.49]$ & $23.1(246)$ & $27.2(155)$ & $18.4(91)$ \\
\hline Widowed & $8.1[0.69]$ & $6.6(70)$ & $10.2(58)$ & $2.4(12)$ \\
\hline Other & - & $2.8(30)$ & $3.9(22)$ & $1.6(8)$ \\
\hline Missing & - & $0.2(2)$ & $0.2(1)$ & $0.2(1)$ \\
\hline \multicolumn{5}{|l|}{ Employment status } \\
\hline Active & $55.7[0.84]$ & $19.0(138)$ & $14.9(85)$ & $10.7(53)$ \\
\hline $\begin{array}{l}\text { Unemployed with } \\
\text { benefits }\end{array}$ & $3.4[0.32]$ & $15.4(164)$ & $13.2(75)$ & $18.0(89)$ \\
\hline $\begin{array}{l}\text { Pensioner or beneficiary } \\
\text { of social help }\end{array}$ & $6.2[0.38]$ & $14.5(154)$ & $15.8(90)$ & $12.9(64)$ \\
\hline $\begin{array}{l}\text { Unemployed without } \\
\text { benefits }\end{array}$ & $2.7[0.28]$ & $57.0(607)$ & $56.0(319)$ & $58.2(288)$ \\
\hline Housewife/husband & $11.0[0.42]$ & na & na & na \\
\hline Student & $3.8[0.32]$ & na & na & na \\
\hline Other & $\mathrm{Na}$ & na & na & na \\
\hline Missing & $0.3[0.09]$ & $0.0(2)$ & $0.2(1)$ & $0.2(1)$ \\
\hline \multicolumn{5}{|l|}{ Number of children } \\
\hline \multicolumn{5}{|c|}{${ }^{\dagger}$ Data from the Catalan Health Survey (Enquesta de Salut de Catalunya (ESCA)) in 2018.} \\
\hline \multicolumn{5}{|c|}{${ }^{\ddagger}$ With the exception of Mean age (mean (SE)). } \\
\hline \multicolumn{5}{|c|}{$\begin{array}{l}\S \text { Data from the Catalan Health Survey (ESCA) in } 2014 \text { (the last year this data was collected) }(n= \\
\text { 1804). }\end{array}$} \\
\hline \multicolumn{5}{|c|}{ PB: Pharmaceutical Bank; na: Data not available or not applicable. } \\
\hline $\begin{array}{l}\text { Bold numbers indicate st } \\
\text { beneficiaries on Students }\end{array}$ & $\begin{array}{l}\text { istically significant d } \\
\text { T test for mean age a }\end{array}$ & $\begin{array}{l}\text { ences }(p<0.05 \\
\text { hi-squared tes }\end{array}$ & $\begin{array}{l}\text { etween male anc } \\
\text { for categorical va }\end{array}$ & $\begin{array}{l}\text { emale } \\
\text { ables. }\end{array}$ \\
\hline
\end{tabular}




\begin{tabular}{|c|c|c|c|c|}
\hline & $\begin{array}{l}\text { General population }^{\dagger} \\
(n=4827) \%[S E]^{\ddagger}\end{array}$ & $\begin{array}{l}\text { PB } \\
\text { beneficiaries } \\
(n=1206) \% \\
(n)^{\ddagger}\end{array}$ & $\begin{array}{l}\text { Female } \\
\text { beneficiaries ( } n \\
=623 \text { ) }\end{array}$ & $\begin{array}{l}\text { Male } \\
\text { beneficiaries } \\
(n=583)\end{array}$ \\
\hline 0 & $\mathrm{Na}$ & $29.1(310)$ & $22.8(130)$ & $36.4(180)$ \\
\hline 1 & $\mathrm{Na}$ & $18.2(194)$ & $20.2(115)$ & $16.0(79)$ \\
\hline 2 & $\mathrm{Na}$ & $26.1(278)$ & $26.8(153)$ & $25.3(125)$ \\
\hline 3 & $\mathrm{Na}$ & 11.9 (127) & $14.6(83)$ & $8.9(44)$ \\
\hline 4 or more children & $\mathrm{Na}$ & $14.7(156)$ & $15.6(89)$ & $13.5(67)$ \\
\hline \multicolumn{5}{|c|}{${ }^{\dagger}$ Data from the Catalan Health Survey (Enquesta de Salut de Catalunya (ESCA)) in 2018.} \\
\hline \multicolumn{5}{|c|}{${ }^{\ddagger}$ With the exception of Mean age (mean (SE)). } \\
\hline \multicolumn{5}{|c|}{$\begin{array}{l}\S \text { Data from the Catalan Health Survey (ESCA) in } 2014 \text { (the last year this data was collected) }(n= \\
\text { 1804). }\end{array}$} \\
\hline \multicolumn{5}{|c|}{ PB: Pharmaceutical Bank; na: Data not available or not applicable. } \\
\hline
\end{tabular}

Compared with the general Catalan population, the population of beneficiaries was older, had a lower level of education, showed a higher prevalence of functional disability, was less likely to be Spanish, and more likely to be divorced and unemployed.

In comparison with male beneficiaries, female beneficiaries were older; more likely to be active and divorced or widowed; and more likely to have children.

Patterns of drugs use

Proportion of beneficiaries with at least one medicine from each group

Tables 2 and 3 show the proportion of beneficiaries using at least one healthcare product or medicine from each anatomical group and therapeutic subgroup (therapeutic subgroups and type of healthcare product are only shown if they have been used by at least $20 \%$ of beneficiaries; full data are shown in supplementary files). This information was not available for the Catalan general population. 
Table 2

Proportion of beneficiaries that received at least one medicine or healthcare product overall and for gender, \% (N).

\begin{tabular}{|c|c|c|c|}
\hline $\begin{array}{l}\text { MEDICINES, Anatomical group (ATC level 1) and therapeutic group } \\
(\text { ATC level 2) }\end{array}$ & $\begin{array}{l}\text { Total } \\
(n= \\
1206)\end{array}$ & $\begin{array}{l}\text { Females } \\
(n= \\
623)\end{array}$ & $\begin{array}{l}\text { Males } \\
(n= \\
583)\end{array}$ \\
\hline Alimentary tract and metabolism (A) & $\begin{array}{l}67.7 \\
(817)\end{array}$ & $\begin{array}{l}73.8 \\
(460)\end{array}$ & $\begin{array}{l}61.2 \\
(357)\end{array}$ \\
\hline Drugs for acid related disorders (A02) & $\begin{array}{l}51.1 \\
(616)\end{array}$ & $\begin{array}{l}55.9 \\
(348)\end{array}$ & $\begin{array}{l}46.0 \\
(268)\end{array}$ \\
\hline Drugs used in diabetes (A10) & $\begin{array}{l}26.0 \\
(314)\end{array}$ & $\begin{array}{l}24.7 \\
(154)\end{array}$ & $\begin{array}{l}27.4 \\
(160)\end{array}$ \\
\hline Blood and blood forming organs (B) & $\begin{array}{l}41.0 \\
(495)\end{array}$ & $\begin{array}{l}38.2 \\
(238)\end{array}$ & $\begin{array}{l}44.1 \\
(257)\end{array}$ \\
\hline Antithrombotic agents (B01) & $\begin{array}{l}30.8 \\
(371)\end{array}$ & $\begin{array}{l}24.4 \\
(152)\end{array}$ & $\begin{array}{l}37.6 \\
(219)\end{array}$ \\
\hline Cardiovascular System (C) & $\begin{array}{l}55.9 \\
(674)\end{array}$ & $\begin{array}{l}53.9 \\
(336)\end{array}$ & $\begin{array}{l}58.0 \\
(338)\end{array}$ \\
\hline Beta blocking agents (C07) & $\begin{array}{l}20.0 \\
(241)\end{array}$ & $\begin{array}{l}13.2 \\
(82)\end{array}$ & $\begin{array}{l}27.3 \\
(159)\end{array}$ \\
\hline Agents acting on the renin-angiotensin system (C09) & $\begin{array}{l}36.0 \\
(434)\end{array}$ & $\begin{array}{l}35.0 \\
(218)\end{array}$ & $\begin{array}{l}37.1 \\
(216)\end{array}$ \\
\hline Lipid modifying agents (C10) & $\begin{array}{l}38.6 \\
(466)\end{array}$ & $\begin{array}{l}32.9 \\
(205)\end{array}$ & $\begin{array}{l}44.8 \\
(261)\end{array}$ \\
\hline Dermatologicals (D) & $\begin{array}{l}20.4 \\
(246)\end{array}$ & $\begin{array}{l}25.0 \\
(156)\end{array}$ & $\begin{array}{l}15.4 \\
(90)\end{array}$ \\
\hline Genito urinary system and sex hormones (G) & $\begin{array}{l}11.4 \\
(137)\end{array}$ & $\begin{array}{l}11.2 \\
(70)\end{array}$ & $\begin{array}{l}11.5 \\
(67)\end{array}$ \\
\hline $\begin{array}{l}\text { Systemic hormonal preparations, excluding sex hormones and } \\
\text { insulins }(H)\end{array}$ & $\begin{array}{l}17.3 \\
(209)\end{array}$ & $\begin{array}{l}23.4 \\
(146)\end{array}$ & $\begin{array}{l}10.8 \\
(63)\end{array}$ \\
\hline Antiinfectives for systemic use $(\mathrm{J})$ & $\begin{array}{l}25.3 \\
(305)\end{array}$ & $\begin{array}{l}28.1 \\
(175)\end{array}$ & $\begin{array}{l}22.3 \\
(130)\end{array}$ \\
\hline Antibacterials for systemic use (J01) & $\begin{array}{l}23.6 \\
(284)\end{array}$ & $\begin{array}{l}26.0 \\
(162)\end{array}$ & $\begin{array}{l}20.9 \\
(122)\end{array}$ \\
\hline
\end{tabular}

\footnotetext{
${ }^{\dagger}$ Only the therapeutic groups and type of healthcare products that have been used by at least a $20 \%$ of the beneficiaries are shown. Supplementary files show full data.

‡For Analgesics and Antiepileptics, which are highly heterogeneous, the Anatomical therapeutic chemical level is shown if $<10 \%$ of the beneficiaries have used medications.
} 


\begin{tabular}{|c|c|c|c|}
\hline $\begin{array}{l}\text { MEDICINES, Anatomical group (ATC level 1) and therapeutic group } \\
(\text { ATC level 2) }\end{array}$ & $\begin{array}{l}\text { Total } \\
(n= \\
1206)\end{array}$ & $\begin{array}{l}\text { Females } \\
(n= \\
623)\end{array}$ & $\begin{array}{l}\text { Males } \\
(n= \\
583)\end{array}$ \\
\hline Antineoplastic and immunomodulating agents $(\mathrm{L})$ & $7.1(86)$ & $\begin{array}{l}10.1 \\
(63)\end{array}$ & $4.0(23)$ \\
\hline Musculo-Skeletal System (M) & $\begin{array}{l}68.1 \\
(821)\end{array}$ & $\begin{array}{l}38.8 \\
(242)\end{array}$ & $\begin{array}{l}24.5 \\
(143)\end{array}$ \\
\hline Antiinflammatory and antirheumatic products (M01) & $\begin{array}{l}26.4 \\
(318)\end{array}$ & $\begin{array}{l}34.0 \\
(212)\end{array}$ & $\begin{array}{l}18.2 \\
(106)\end{array}$ \\
\hline Nervous System (N) & $\begin{array}{l}78.9 \\
(952)\end{array}$ & $\begin{array}{l}84.3 \\
(525)\end{array}$ & $\begin{array}{l}73.2 \\
(427)\end{array}$ \\
\hline Analgesics $(\mathrm{N} 02)^{\ddagger}$ & $\begin{array}{l}56.0 \\
(675)\end{array}$ & $\begin{array}{l}65.8 \\
(410)\end{array}$ & $\begin{array}{l}45.5 \\
(265)\end{array}$ \\
\hline Other opioids (N02AX) & $\begin{array}{l}17.1 \\
(206)\end{array}$ & $\begin{array}{l}22.0 \\
(137)\end{array}$ & $\begin{array}{l}11.8 \\
(69)\end{array}$ \\
\hline Pyrazolones (N02BB) & $\begin{array}{l}18.9 \\
(228)\end{array}$ & $\begin{array}{l}23.9 \\
(149)\end{array}$ & $\begin{array}{l}13.6 \\
(79)\end{array}$ \\
\hline Anilides (N02BE) & $\begin{array}{l}44.4 \\
(535)\end{array}$ & $\begin{array}{l}51.4 \\
(320)\end{array}$ & $\begin{array}{l}36.9 \\
(215)\end{array}$ \\
\hline Antiepileptics (N03) & $\begin{array}{l}26.5 \\
(319)\end{array}$ & $\begin{array}{l}29.1 \\
(181)\end{array}$ & $\begin{array}{l}23.7 \\
(138)\end{array}$ \\
\hline Other antiepileptics (N03AX) & $\begin{array}{l}22.2 \\
(268)\end{array}$ & $\begin{array}{l}26.0 \\
(162)\end{array}$ & $\begin{array}{l}18.2 \\
(106)\end{array}$ \\
\hline Psycholeptics (N05) & $\begin{array}{l}43.5 \\
(525)\end{array}$ & $\begin{array}{l}46.7 \\
(291)\end{array}$ & $\begin{array}{l}40.1 \\
(234)\end{array}$ \\
\hline Psychoanaleptics (N06) & $\begin{array}{l}41.9 \\
(505)\end{array}$ & $\begin{array}{l}49.1 \\
(306)\end{array}$ & $\begin{array}{l}34.1 \\
(199)\end{array}$ \\
\hline Antiparasitic products, insecticides and repellents $(P)$ & $1.6(19)$ & $1.8(11)$ & $1.4(8)$ \\
\hline Respiratory System (R) & $\begin{array}{l}32.1 \\
(387)\end{array}$ & $\begin{array}{l}34.0 \\
(212)\end{array}$ & $\begin{array}{l}30.0 \\
(175)\end{array}$ \\
\hline Drugs for obstructive airway diseases (R03) & $\begin{array}{l}21.3 \\
(257)\end{array}$ & $\begin{array}{l}21.4 \\
(133)\end{array}$ & $\begin{array}{l}21.3 \\
(124)\end{array}$ \\
\hline Sensory Organs (S) & $\begin{array}{l}13.4 \\
(161)\end{array}$ & $\begin{array}{l}15.9 \\
(99)\end{array}$ & $\begin{array}{l}10.6 \\
(62)\end{array}$ \\
\hline
\end{tabular}

${ }^{\dagger}$ Only the therapeutic groups and type of healthcare products that have been used by at least a $20 \%$ of the beneficiaries are shown. Supplementary files show full data.

₹ For Analgesics and Antiepileptics, which are highly heterogeneous, the Anatomical therapeutic chemical level is shown if $<10 \%$ of the beneficiaries have used medications. 


\begin{tabular}{|c|c|c|c|}
\hline $\begin{array}{l}\text { MEDICINES, Anatomical group (ATC level 1) and therapeutic group } \\
(\text { ATC level 2) }\end{array}$ & $\begin{array}{l}\text { Total } \\
\\
(n= \\
1206)\end{array}$ & $\begin{array}{l}\text { Females } \\
(n= \\
623)\end{array}$ & $\begin{array}{l}\text { Males } \\
(n= \\
583)\end{array}$ \\
\hline Various (V) & $1.8(22)$ & $2.1(13)$ & $1.5(9)$ \\
\hline HEALTHCARE PRODUCTS & $\begin{array}{l}18.7 \\
(226)\end{array}$ & $\begin{array}{l}20.7 \\
(129)\end{array}$ & $\begin{array}{l}16.6 \\
(97)\end{array}$ \\
\hline \multicolumn{4}{|c|}{$\begin{array}{l}\text { + } \text { Only the therapeutic groups and type of healthcare products that have been used by at least a } 20 \% \\
\text { of the beneficiaries are shown. Supplementary files show full data. }\end{array}$} \\
\hline
\end{tabular}


Table 3

Proportion of beneficiaries that received at least one medicine or healthcare product for age groups, \% (N).

\section{MEDICINES, Anatomical group (ATC level 1) and therapeutic group (ATC level 2) ${ }^{\dagger}$}

Alimentary tract and metabolism (A)

Drugs for acid related disorders (A02)

Drugs used in diabetes (A10)

Blood and blood forming organs (B)

Antithrombotic agents (B01)

Cardiovascular System (C)

Diuretics (C03)

Beta blocking agents (C07)

Calcium channel blockers (C08)

Agents acting on the renin-angiotensin system (C09)

Lipid modifying agents (C10)

Dermatologicals (D)

Genito urinary system and sex hormones $(G)$

Systemic hormonal preparations, excl. Sex hormones and insulins $(\mathrm{H})$

Antiinfectives for systemic use (J)

Antibacterials for systemic use (J01)

Antineoplastic and immunomodulating agents $(\mathrm{L})$

Musculo-Skeletal System (M)

Antiinflammatory and antirheumatic products (M01)

\begin{tabular}{|c|c|}
\hline$\underset{104)}{\leq 14}(n=$ & $\begin{array}{l}15-64(n= \\
918)\end{array}$ \\
\hline
\end{tabular}

$13.3(14) \quad 70.6(647)$

84.8

(156)

5.7 (6) $\quad 53.5(491)$

64.7

$1.0(1) \quad 25.6(235) \quad 42.4(78)$

$7.6(8) \quad 41.3(379)$

58.7

$0(0) \quad 30.2(277) \quad 51.2(94)$

$9.5(10) \quad 55.6(510)$

83.7

$0(0)$

$14.5(133)$

$25.5(47)$

$2.9(3)$

20.6 (189)

$26.6(49)$

$1.0(1)$

$13.4(123)$

$25.0(46)$

$1.9(2)$

34.6 (317)

62.5

(115)

$0(0)$

$38.6(354)$

60.9

(112)

$12.4(13) \quad 20.5(188) \quad 24.5(45)$

$1.9(2) \quad 10.9(100) \quad 19.0(35)$

$10.5(11) \quad 17.9(164) \quad 18.5(34)$

$19.1(20) \quad 26.7(245) \quad 21.7(40)$

$18.1(19) \quad 24.9(229) \quad 19.9(36)$

$5.7(6) \quad 7.2(66) \quad 7.6(14)$

$21.9(23) \quad 34.6(317) \quad 24.5(45)$

$21.0(22) \quad 28.6(262) \quad 18.5(34)$

${ }^{\dagger}$ Only the therapeutic groups and type of healthcare products that have been used by at least a $20 \%$ of the beneficiaries are shown. Supplementary files show full data.

‡ For Analgesics and Antiepileptics, which are highly heterogeneous, the Anatomical therapeutic chemical level is shown if $<10 \%$ of the beneficiaries have used medications. 


\begin{tabular}{|c|c|c|c|}
\hline $\begin{array}{l}\text { MEDICINES, Anatomical group (ATC level 1) and therapeutic } \\
\text { group (ATC level 2) }\end{array}$ & $\begin{array}{l}\leq 14(n= \\
104)\end{array}$ & $\begin{array}{l}15-64(n= \\
918)\end{array}$ & $\geq 65(n=$ \\
\hline Nervous System (N) & $61.0(64)$ & $80.3(736)$ & $\begin{array}{l}82.6 \\
(152)\end{array}$ \\
\hline Analgesics (N02) ${ }^{\ddagger}$ & $22.9(24)$ & $58.2(534)$ & $\begin{array}{l}63.6 \\
(117)\end{array}$ \\
\hline Other opioids (N02AX) & 0 & $19.1(175)$ & $16.6(31)$ \\
\hline Pyrazolones (N02BB) & 0 & $20.2(185)$ & $23.0(43)$ \\
\hline Anilides (N02BE) & $21.2(22)$ & $44.7(409)$ & $\begin{array}{l}55.6 \\
(104)\end{array}$ \\
\hline Antiepileptics (N03)\& & $9.5(10)$ & $30.0(275)$ & $18.5(34)$ \\
\hline Other antiepileptics (N03AX) & $3.9(4)$ & $25.4(232)$ & $17.1(32)$ \\
\hline Psycholeptics (N05) & $28.6(30)$ & $46.1(423)$ & $39.1(72)$ \\
\hline Psychoanaleptics (N06) & $33.3(35)$ & $43.4(398)$ & $39.1(72)$ \\
\hline Antiparasitic products, insecticides and repellents $(P)$ & $2.9(3)$ & $1.7(16)$ & $0(0)$ \\
\hline Respiratory System (R) & $36.2(38)$ & $31.5(289)$ & $32.6(60)$ \\
\hline Drugs for obstructive airway diseases (R03) & $26.7(28)$ & $20.0(183)$ & $25.0(46)$ \\
\hline Antihistamines for systemic use (R06) & $20.0(21)$ & $13.6(125)$ & $9.8(18)$ \\
\hline Sensory Organs (S) & $12.4(13)$ & $11.2(103)$ & $24.5(45)$ \\
\hline Ophthalmologicals (S01) & $11.4(12)$ & $9.7(89)$ & $23.8(43)$ \\
\hline Various (V) & $0(0)$ & $2.2(20)$ & $1.1(2)$ \\
\hline HEALTHCARE PRODUCTS & $38.1(40)$ & $14.4(132)$ & $29.4(54)$ \\
\hline Diapers & $10.5(11)$ & $4.8(44)$ & $21.2(39)$ \\
\hline Allergen-specific immunotherapy & $21.0(22)$ & $1.4(13)$ & $0(0)$ \\
\hline \multicolumn{4}{|c|}{$\begin{array}{l}+ \text { Only the therapeutic groups and type of healthcare products that have been used by at least a } 20 \% \\
\text { of the beneficiaries are shown. Supplementary files show full data. }\end{array}$} \\
\hline
\end{tabular}

The medications most commonly consumed by the beneficiaries were those related to the 'nervous system' (78.9\%), followed by those related to the 'musculo-skeletal system' (68.1\%), 'alimentary tract and metabolism' (67.7\%) and 'cardiovascular system' (55.9\%). Disaggregation by therapeutic subgroup indicated that a large proportion of the population received medication for pain-related disorders 
[analgesics (56.0\%); anti-inflammatory and antirheumatic products (26.4\%) and other antiepileptics (22.2\%)], gastrointestinal disorders [drugs for acid-related disorders (51.1\%) and drugs for functional gastrointestinal disorders (7.6\%)], mental health related disorders [psycholeptics (43.5\%) and psychoanaleptics (41.9\%)], cardiovascular diseases [lipid modifying agents (38.6\%), agents acting on the renin-angiotensin system $(36.0 \%)$, antithrombotic agents $(30.8 \%)$, beta blocking agents $(20.0 \%)$, diuretics (14.9\%), calcium channel blockers (14.1\%)], diabetes [drugs used in diabetes $(26.0 \%)$ ] and allergies and asthma [drugs for obstructive airway diseases (21.3\%) and antihistamines for systemic use (13.6\%)]. Almost $19 \%$ of NGO beneficiaries used at least one healthcare product.

Males and females followed a similar pattern (Table 2) although, compared with male beneficiaries, females were more likely to use medication for pain-related disorders (e.g., $65.8 \%$ vs. $45.5 \%$ in analgesics), gastrointestinal disorders (e.g., $55.9 \%$ vs. $46.0 \%$ in drugs for acid related disorders) and mental health disorders (e.g., $49.1 \%$ vs. $34.1 \%$ in psychoanaleptics), and less likely to use medications for cardiovascular diseases (e.g., $32.9 \%$ vs. $44.8 \%$ in lipid modifying agents) and diabetes (e.g., $24.7 \%$ vs. $27.4 \%$ in drugs used in diabetes).

Among children (Table 3), the most frequently used medicines were those for the 'nervous system' (61.0\%), the 'respiratory system' (36.2\%) and the 'musculo-skeletal system' (21.9\%). There was a highly prevalent use of medicines for the 'nervous system' (80.3\%), 'alimentary tract and metabolism' (70.6\%) and 'cardiovascular system' (55.6\%) among beneficiaries aged between 15 and 64 years old. Over $80 \%$ of the oldest group of beneficiaries was using medications for the 'alimentary tract and metabolism', 'cardiovascular system' and 'nervous system'.

\section{Volume of medicines and healthcare products covered by the PB and the CatSalut}

Tables 4 and 5 show the relative weight of each medicine and healthcare product with respect to the total number of units of medicines and healthcare products, for PB beneficiaries and for the whole Catalan population. 
Table 4

Proportion of medicines and products used by the beneficiaries and the general population, \%(N).

Total

PB Catalonia

48747
Males

PB Catalonia PB

2122559400000
Females

PB Catalonia

$27522 \quad 77500000$

\section{Anatomical group \\ (ATC level 1) and therapeutic group $\left(\right.$ ATC level 2) ${ }^{\dagger}$}

Alimentary tract and metabolism (A)

Drugs for acid related disorders (A02)

Drugs used in diabetes (A10)

Blood and blood

forming organs (B)

Cardiovascular

System (C)

Lipid modifying

agents (C10)

Dermatologicals (D)

Genito urinary system $\quad 1.4$

and sex hormones (G) (668)

Systemic hormonal $\quad 1.5$

preparations, excl. Sex

hormones and

insulins $(\mathrm{H})$

Antiinfectives for systemic use $(J)$

Antineoplastic and immunomodulating agents $(\mathrm{L})$

Musculo-Skeletal

System (M)

3.9

(1878)

\section{0}

(7788)

14.3

(19644551)

\section{7}

(2777)

6.2

(8547897)

6.3

(3081)

4.9

\section{3}

(3052)

6.1

(8310079)

19.1

(9320)

25.0

(34405442)

5.8

(2836)

6.6

(9019823)

$\begin{array}{ll}2.0 & 1.7 \\ (951) & (23687\end{array}$

(2368761)

(720)

2.7

(3769794)

1.8

(2510611)
(6777457)

\section{9}

(3375)

14.5

(8632772)

$5.4 \quad 6.0$

(1136)

\section{0}

$\begin{array}{ll}7.6 & 6.4 \\ (1621) & (38144\end{array}$

(1621)

8.1

(1711)

7.0

(4163092)

24.9

(5281)

29.0

(17200000)

7.7

(1636)

8.0

(4767132)

1.4

(304)

1.8

(1081384)

1.7

(369)

4.3

(2538678)

0.7

(146)

1.3

(763523)

$2.1 \quad 2.2$

(574)

4.4

(1200)

2.4

(647)

1.1

(299)

(1219365)

1.7
$(1274461)$

1.6

$2.1 \quad 2.2$

(1736597)

14.1

6.4

(4950959)

3.8

(2945215)

5.3

22.1

5.5

(4229100)

1.7

.6
(10900000)

(4123536)

(17100000)

$(574)$

3.3

$\begin{array}{lll}2.2 & 3.3 & 2.8\end{array}$

(473) (1942406) (774)

3.4

(1247)

3.4
$(4598444)$

$1.7 \quad 0.9$

(369)

(556179)

2.4

(660)

1.0

(804895)

(1364320)

1.5

3.0

4.4

4.5

4.7

(2085977)

(644)

(2619746)

(1234)

$(3635542)$

${ }^{\dagger}$ The table only shows the therapeutic groups and type of healthcare products that represent at least a $5 \%$ of the total volume of medications and healthcare products, respectively. The whole table can be consulted in Supplementary Table 1.

na $=$ not available. 


\begin{tabular}{|c|c|c|c|c|c|c|}
\hline & Total & & Males & & Females & \\
\hline Nervous System (N) & $\begin{array}{l}36.9 \\
(17985)\end{array}$ & $\begin{array}{l}27.3 \\
(37443242)\end{array}$ & $\begin{array}{l}32.2 \\
(6835)\end{array}$ & $\begin{array}{l}21.3 \\
(12600000)\end{array}$ & $\begin{array}{l}40.5 \\
(11150)\end{array}$ & $\begin{array}{l}31.9 \\
(24700000)\end{array}$ \\
\hline Analgesics (N02) & $\begin{array}{l}14.1 \\
(6848)\end{array}$ & $\begin{array}{l}9.7 \\
(13364618)\end{array}$ & $\begin{array}{l}11.0 \\
(2328)\end{array}$ & $\begin{array}{l}7.1 \\
(4212587)\end{array}$ & $\begin{array}{l}16.4 \\
(4520)\end{array}$ & $\begin{array}{l}11.8 \\
(9107326)\end{array}$ \\
\hline Antiepileptics (N03) & $\begin{array}{l}5.1 \\
(2461)\end{array}$ & $\begin{array}{l}2.3 \\
(3124887)\end{array}$ & $\begin{array}{l}4.8 \\
(1018)\end{array}$ & $\begin{array}{l}2.3 \\
(1354867)\end{array}$ & $\begin{array}{l}5.2 \\
(1443)\end{array}$ & $\begin{array}{l}2.3 \\
(1758064)\end{array}$ \\
\hline Psycholeptics (N05) & $\begin{array}{l}9.4 \\
(4563)\end{array}$ & $\begin{array}{l}8.5 \\
(11614648)\end{array}$ & $\begin{array}{l}9.3 \\
(1975)\end{array}$ & $\begin{array}{l}6.9 \\
(4096660)\end{array}$ & $\begin{array}{l}9.4 \\
(2588)\end{array}$ & $\begin{array}{l}9.7 \\
(7481897)\end{array}$ \\
\hline $\begin{array}{l}\text { Psychoanaleptics } \\
\text { (N06) }\end{array}$ & $\begin{array}{l}7.1 \\
(3472)\end{array}$ & $\begin{array}{l}5.6 \\
(7703802)\end{array}$ & $\begin{array}{l}6.1 \\
(1302)\end{array}$ & $\begin{array}{l}3.9 \\
(2296327)\end{array}$ & $\begin{array}{l}7.9 \\
(2170)\end{array}$ & $\begin{array}{l}7.0 \\
(5387490)\end{array}$ \\
\hline $\begin{array}{l}\text { Antiparasitic products, } \\
\text { insecticides and } \\
\text { repellents }(P)\end{array}$ & $\begin{array}{l}0.3 \\
(134)\end{array}$ & $\begin{array}{l}0.2 \\
(301954)\end{array}$ & $\begin{array}{l}0.4 \\
(84)\end{array}$ & $\begin{array}{l}0.2 \\
(101466)\end{array}$ & $0.2(50)$ & $\begin{array}{l}0.3 \\
(198061)\end{array}$ \\
\hline $\begin{array}{l}\text { Respiratory System } \\
\text { (R) }\end{array}$ & $\begin{array}{l}6.3 \\
(3084)\end{array}$ & $\begin{array}{l}5.5 \\
(7577337)\end{array}$ & $\begin{array}{l}6.2 \\
(1323)\end{array}$ & $\begin{array}{l}6.2 \\
(3661700)\end{array}$ & $\begin{array}{l}6.4 \\
(1761)\end{array}$ & $\begin{array}{l}5.0 \\
(3882757)\end{array}$ \\
\hline Sensory Organs (S) & $\begin{array}{l}1.6 \\
(793)\end{array}$ & $\begin{array}{l}6.2 \\
(8549307)\end{array}$ & $\begin{array}{l}1.2 \\
(251)\end{array}$ & $\begin{array}{l}5.7 \\
(3386436)\end{array}$ & $\begin{array}{l}2.0 \\
(542)\end{array}$ & $\begin{array}{l}6.6 \\
(5136004)\end{array}$ \\
\hline Various (V) & $0.2(98)$ & $\begin{array}{l}0.1 \\
(159982)\end{array}$ & $\begin{array}{l}0.3 \\
(60)\end{array}$ & $0.2(86633)$ & $0.1(38)$ & $0.1(73017)$ \\
\hline $\begin{array}{l}\text { HEALTHCARE } \\
\text { PRODUCTS }\end{array}$ & 2276 & na & 972 & na & 1304 & na \\
\hline Wound dressings & $\begin{array}{l}47.4 \\
(1079)\end{array}$ & na & $\begin{array}{l}47.5 \\
(462)\end{array}$ & na & $\begin{array}{l}47.3 \\
(617)\end{array}$ & na \\
\hline $\begin{array}{l}\text { Surgical wound } \\
\text { dressings }\end{array}$ & $\begin{array}{l}20.0 \\
(456)\end{array}$ & na & $\begin{array}{l}27.9 \\
(271)\end{array}$ & na & $\begin{array}{l}14.2 \\
(185)\end{array}$ & na \\
\hline Diapers & $\begin{array}{l}27.9 \\
(636)\end{array}$ & na & $\begin{array}{l}20.4 \\
(198)\end{array}$ & na & $\begin{array}{l}33.6 \\
(438)\end{array}$ & na \\
\hline \multicolumn{7}{|c|}{$\begin{array}{l}{ }^{\dagger} \text { The table only shows the therapeutic groups and type of healthcare products that represent at least } \\
\text { a } 5 \% \text { of the total volume of medications and healthcare products, respectively. The whole table can be } \\
\text { consulted in Supplementary Table } 1 .\end{array}$} \\
\hline th & & & & & & \\
\hline
\end{tabular}


Table 5

Proportion of medicines and healthcare products used by age group, \% (N).

\begin{tabular}{|c|c|c|c|c|c|c|}
\hline & \multicolumn{2}{|l|}{$\leq 14$} & \multicolumn{2}{|l|}{$15-64$} & \multicolumn{2}{|l|}{$\geq 65$} \\
\hline & PB & Catalonia & PB & Catalonia & PB & Catalonia \\
\hline $\begin{array}{l}\text { MEDICINES, Anatomical } \\
\text { group (ATC level 1) and } \\
\text { therapeutic group (ATC } \\
\text { level 2) }\end{array}$ & $\begin{array}{l}N= \\
2417\end{array}$ & $\begin{array}{l}N= \\
3892497\end{array}$ & $\begin{array}{l}N= \\
40378\end{array}$ & $\begin{array}{l}N= \\
50320043\end{array}$ & $\begin{array}{l}N= \\
8228\end{array}$ & $\begin{array}{l}N= \\
82689934\end{array}$ \\
\hline $\begin{array}{l}\text { Alimentary tract and } \\
\text { metabolism }(A)\end{array}$ & $\begin{array}{l}11.3 \\
(235)\end{array}$ & $\begin{array}{l}5.22 \\
(203126)\end{array}$ & $\begin{array}{l}15.8 \\
(6161)\end{array}$ & $\begin{array}{l}13.0 \\
(6533202)\end{array}$ & $\begin{array}{l}17.9 \\
(1392)\end{array}$ & $\begin{array}{l}15.5 \\
(12800000)\end{array}$ \\
\hline $\begin{array}{l}\text { Drugs for acid related } \\
\text { disorders (A02) }\end{array}$ & $\begin{array}{l}0.9 \\
(18)\end{array}$ & $\begin{array}{l}0.37 \\
(14432)\end{array}$ & $\begin{array}{l}5.9 \\
(2279)\end{array}$ & $\begin{array}{l}5.2 \\
(2596489)\end{array}$ & $\begin{array}{l}6.2 \\
(480)\end{array}$ & $\begin{array}{l}7.2 \\
(5911230)\end{array}$ \\
\hline $\begin{array}{l}\text { Drugs used in diabetes } \\
\text { (A10) }\end{array}$ & $0.2(4)$ & $\begin{array}{l}0.53 \\
(20824)\end{array}$ & $\begin{array}{l}6.2 \\
(2410)\end{array}$ & $\begin{array}{l}4.7 \\
(2342833)\end{array}$ & $\begin{array}{l}8.6 \\
(667)\end{array}$ & $\begin{array}{l}5.3 \\
(4395978)\end{array}$ \\
\hline $\begin{array}{l}\text { Other alimentary tract and } \\
\text { metabolism products } \\
\text { (A16) }\end{array}$ & $\begin{array}{l}5.7 \\
(120)\end{array}$ & $\begin{array}{l}0.15 \\
(5962)\end{array}$ & $0(0)$ & $\begin{array}{l}<0.1 \\
(9889)\end{array}$ & $0(0)$ & $\begin{array}{l}<0.1 \\
(2762)\end{array}$ \\
\hline $\begin{array}{l}\text { Blood and blood forming } \\
\text { organs (B) }\end{array}$ & $\begin{array}{l}0.8 \\
(16)\end{array}$ & $\begin{array}{l}1.59 \\
(61871)\end{array}$ & $\begin{array}{l}6.0 \\
(2315)\end{array}$ & $\begin{array}{l}4.4 \\
(2201112)\end{array}$ & $\begin{array}{l}9.3 \\
(721)\end{array}$ & $\begin{array}{l}7.3 \\
(6023645)\end{array}$ \\
\hline $\begin{array}{l}\text { Antithrombotic agents } \\
\text { (B01) }\end{array}$ & $0(0)$ & $\begin{array}{l}0.1 \\
(3948)\end{array}$ & $\begin{array}{l}4.3 \\
(1664)\end{array}$ & $\begin{array}{l}2.8 \\
(1397537)\end{array}$ & $\begin{array}{l}6.5 \\
(507)\end{array}$ & $\begin{array}{l}6.0 \\
(4945210)\end{array}$ \\
\hline Cardiovascular System (C) & $\begin{array}{l}5.7 \\
(120)\end{array}$ & $\begin{array}{l}0.89 \\
(34789)\end{array}$ & $\begin{array}{l}18.7 \\
(7286)\end{array}$ & $\begin{array}{l}19.2 \\
(9654331)\end{array}$ & $\begin{array}{l}24.7 \\
(1914)\end{array}$ & $\begin{array}{l}29.8 \\
(24600000)\end{array}$ \\
\hline $\begin{array}{l}\text { Agents acting on the renin- } \\
\text { angiotensin system (CO9) }\end{array}$ & $\begin{array}{l}2.2 \\
(46)\end{array}$ & $\begin{array}{l}0.1 \\
(3756)\end{array}$ & $\begin{array}{l}4.6 \\
(1806)\end{array}$ & $\begin{array}{l}7.0 \\
(3526984)\end{array}$ & $\begin{array}{l}7.2 \\
(559)\end{array}$ & $\begin{array}{l}9.5 \\
(7857509)\end{array}$ \\
\hline Dermatologicals (D) & $\begin{array}{l}2.9 \\
(60)\end{array}$ & $\begin{array}{l}6.34 \\
(246778)\end{array}$ & $\begin{array}{l}2.0 \\
(768)\end{array}$ & $\begin{array}{l}2.2 \\
(1117447)\end{array}$ & $\begin{array}{l}1.6 \\
(123)\end{array}$ & $\begin{array}{l}1.2 \\
(991620)\end{array}$ \\
\hline $\begin{array}{l}\text { Genito urinary system and } \\
\text { sex hormones }(G)\end{array}$ & $0.4(8)$ & $\begin{array}{l}0.22 \\
(8437)\end{array}$ & $\begin{array}{l}1.2 \\
(481)\end{array}$ & $\begin{array}{l}2.8 \\
(1403516)\end{array}$ & $\begin{array}{l}2.3 \\
(179)\end{array}$ & $\begin{array}{l}2.8 \\
(2346090)\end{array}$ \\
\hline $\begin{array}{l}\text { Systemic hormonal } \\
\text { preparations, excl. Sex } \\
\text { hormones and insulins }(H)\end{array}$ & $\begin{array}{l}2.1 \\
(43)\end{array}$ & $\begin{array}{l}5.58 \\
(217191)\end{array}$ & $\begin{array}{l}1.4 \\
(558)\end{array}$ & $\begin{array}{l}2.4 \\
(1180885)\end{array}$ & $\begin{array}{l}1.5 \\
(119)\end{array}$ & $\begin{array}{l}1.3 \\
(1102044)\end{array}$ \\
\hline $\begin{array}{l}\text { Antiinfectives for systemic } \\
\text { use }(J)\end{array}$ & $\begin{array}{l}6.2 \\
(129)\end{array}$ & $\begin{array}{l}17.19 \\
(668951)\end{array}$ & $\begin{array}{l}2.6 \\
(1012)\end{array}$ & $\begin{array}{l}4.6 \\
(2332305)\end{array}$ & $\begin{array}{l}1.4 \\
(106)\end{array}$ & $\begin{array}{l}1.9 \\
(1552050)\end{array}$ \\
\hline
\end{tabular}

${ }^{\dagger}$ The table only shows the therapeutic groups and type of healthcare products that represent at least a $5 \%$ of the total volume of medications and healthcare products, respectively. The whole table can be consulted in Supplementary Table 2. 


\begin{tabular}{|c|c|c|c|c|c|c|}
\hline & $\leq 14$ & & $15-64$ & & $\geq 65$ & \\
\hline $\begin{array}{l}\text { Antibacterials for systemic } \\
\text { use (J01) }\end{array}$ & $\begin{array}{l}5.1 \\
(107)\end{array}$ & $\begin{array}{l}16.56 \\
(644641)\end{array}$ & $\begin{array}{l}2.0 \\
(759)\end{array}$ & $\begin{array}{l}4.2 \\
(2087746)\end{array}$ & $\begin{array}{l}1.2 \\
(93)\end{array}$ & $\begin{array}{l}1.8 \\
(1448848)\end{array}$ \\
\hline $\begin{array}{l}\text { Antineoplastic and } \\
\text { immunomodulating } \\
\text { agents }(L)\end{array}$ & $\begin{array}{l}5.0 \\
(105)\end{array}$ & $\begin{array}{l}0.71 \\
(27603)\end{array}$ & $\begin{array}{l}2.3 \\
(878)\end{array}$ & $\begin{array}{l}1.5 \\
(743741)\end{array}$ & $\begin{array}{l}0.6 \\
(46)\end{array}$ & $\begin{array}{l}0.7 \\
(589730)\end{array}$ \\
\hline $\begin{array}{l}\text { Immunosuppressants } \\
\text { (L04) }\end{array}$ & $\begin{array}{l}5.0 \\
(105)\end{array}$ & $\begin{array}{l}0.48 \\
(18616)\end{array}$ & $\begin{array}{l}2.2 \\
(841)\end{array}$ & $\begin{array}{l}1.1 \\
(552983)\end{array}$ & $\begin{array}{l}0.3 \\
(20)\end{array}$ & $\begin{array}{l}0.4 \\
(310146)\end{array}$ \\
\hline $\begin{array}{l}\text { Musculo-Skeletal System } \\
\text { (M) }\end{array}$ & $\begin{array}{l}3.3 \\
(69)\end{array}$ & $\begin{array}{l}11.91 \\
(463423)\end{array}$ & $\begin{array}{l}4.2 \\
(1651)\end{array}$ & $\begin{array}{l}6.9 \\
(3487078)\end{array}$ & $\begin{array}{l}2.0 \\
(158)\end{array}$ & $\begin{array}{l}2.8 \\
(2304787)\end{array}$ \\
\hline Nervous System (N) & $\begin{array}{l}48.0 \\
(1004)\end{array}$ & $\begin{array}{l}18.7 \\
(727716)\end{array}$ & $\begin{array}{l}37.8 \\
(14713)\end{array}$ & $\begin{array}{l}32.6 \\
(16400000)\end{array}$ & $\begin{array}{l}29.2 \\
(2268)\end{array}$ & $\begin{array}{l}24.4 \\
(20200000)\end{array}$ \\
\hline Analgesics (N02) & $\begin{array}{l}2.91 \\
(61)\end{array}$ & $\begin{array}{l}9.52 \\
(370691)\end{array}$ & $\begin{array}{l}14.5 \\
(5644)\end{array}$ & $\begin{array}{l}8.7 \\
(4400211)\end{array}$ & $\begin{array}{l}14.7 \\
(1143)\end{array}$ & $\begin{array}{l}10.3 \\
(8549011)\end{array}$ \\
\hline Antiepileptics (N03) & $\begin{array}{l}9.8 \\
(206)\end{array}$ & $\begin{array}{l}2.27 \\
(88513)\end{array}$ & $\begin{array}{l}5.3 \\
(2062)\end{array}$ & $\begin{array}{l}3.7 \\
(1834870)\end{array}$ & $\begin{array}{l}2.5 \\
(193)\end{array}$ & $\begin{array}{l}1.4 \\
(1189548)\end{array}$ \\
\hline $\begin{array}{l}\text { Anti-parkinson drugs } \\
\text { (N04) }\end{array}$ & $\begin{array}{l}5.1 \\
(107)\end{array}$ & $\begin{array}{l}0.07 \\
(2687)\end{array}$ & $0.1(51)$ & $\begin{array}{l}0.3 \\
(171035)\end{array}$ & $\begin{array}{l}0.3 \\
(21)\end{array}$ & $\begin{array}{l}0.6 \\
(489661)\end{array}$ \\
\hline Psycholeptics (N05) & $\begin{array}{l}15.0 \\
(314)\end{array}$ & $\begin{array}{l}2.56 \\
(99491)\end{array}$ & $\begin{array}{l}9.8 \\
(3804)\end{array}$ & $\begin{array}{l}11.7 \\
(5888111)\end{array}$ & $\begin{array}{l}5.7 \\
(445)\end{array}$ & $\begin{array}{l}6.8 \\
(5590955)\end{array}$ \\
\hline Psychoanaleptics (N06) & $\begin{array}{l}14.5 \\
(303)\end{array}$ & $\begin{array}{l}4.1 \\
(159702)\end{array}$ & $\begin{array}{l}7.2 \\
(2794)\end{array}$ & $\begin{array}{l}7.5 \\
(3761175)\end{array}$ & $\begin{array}{l}4.8 \\
(375)\end{array}$ & $\begin{array}{l}4.6 \\
(3762940)\end{array}$ \\
\hline $\begin{array}{l}\text { Antiparasitic products, } \\
\text { insecticides and repellents } \\
\text { (P) }\end{array}$ & $0.2(4)$ & $\begin{array}{l}1.16 \\
(45153)\end{array}$ & $\begin{array}{l}0.3 \\
(130)\end{array}$ & $\begin{array}{l}0.4 \\
(193986)\end{array}$ & $0(0)$ & $0.1(60388)$ \\
\hline Respiratory System (R) & $\begin{array}{l}12.7 \\
(265)\end{array}$ & $\begin{array}{l}15.46 \\
(601758)\end{array}$ & $\begin{array}{l}6.3 \\
(2462)\end{array}$ & $\begin{array}{l}6.5 \\
(3272162)\end{array}$ & $\begin{array}{l}4.6 \\
(357)\end{array}$ & $\begin{array}{l}4.4 \\
(3670537)\end{array}$ \\
\hline $\begin{array}{l}\text { Drugs for obstructive } \\
\text { airway diseases (R03) }\end{array}$ & $\begin{array}{l}7.4 \\
(154)\end{array}$ & $\begin{array}{l}10.12 \\
(393914)\end{array}$ & $\begin{array}{l}4.0 \\
(1544)\end{array}$ & $\begin{array}{l}3.2 \\
(1622557)\end{array}$ & $\begin{array}{l}3.5 \\
(268)\end{array}$ & $\begin{array}{l}3.3 \\
(2714370)\end{array}$ \\
\hline Sensory Organs (S) & $\begin{array}{l}1.7 \\
(35)\end{array}$ & $\begin{array}{l}14.37 \\
(559340)\end{array}$ & $\begin{array}{l}1.1 \\
(412)\end{array}$ & $\begin{array}{l}3.4 \\
(1715146)\end{array}$ & $\begin{array}{l}4.5 \\
(346)\end{array}$ & $\begin{array}{l}7.6 \\
(6247954)\end{array}$ \\
\hline Various (V) & $0(0)$ & $\begin{array}{l}0.22 \\
(8459)\end{array}$ & $0.2(67)$ & $0.1(58570)$ & $\begin{array}{l}0.4 \\
(31)\end{array}$ & $0.1(92621)$ \\
\hline HEALTHCARE PRODUCTS & $\begin{array}{l}N= \\
324\end{array}$ & & $\begin{array}{l}N= \\
1484\end{array}$ & & $\begin{array}{l}N= \\
468\end{array}$ & \\
\hline \multicolumn{7}{|c|}{$\begin{array}{l}{ }^{+} \text {The table only shows the therapeutic groups and type of healthcare products that represent at least } \\
\text { a } 5 \% \text { of the total volume of medications and healthcare products, respectively. The whole table can be } \\
\text { consulted in Supplementary Table } 2 \text {. }\end{array}$} \\
\hline na: not available & & & & & & \\
\hline
\end{tabular}




\begin{tabular}{|c|c|c|c|c|c|c|}
\hline & $\leq 14$ & & $15-64$ & & $\geq 65$ & \\
\hline Wound dressings & $\begin{array}{l}12.0 \\
(39)\end{array}$ & na & $\begin{array}{l}58.2 \\
(863)\end{array}$ & na & $\begin{array}{l}37.82 \\
(177)\end{array}$ & na \\
\hline Surgical wound dressings & $\begin{array}{l}39.2 \\
(127)\end{array}$ & na & $\begin{array}{l}18.9 \\
(280)\end{array}$ & na & $\begin{array}{l}10.47 \\
(49)\end{array}$ & na \\
\hline Diapers & $\begin{array}{l}39.2 \\
(127)\end{array}$ & na & $\begin{array}{l}18.7 \\
(277)\end{array}$ & na & $\begin{array}{l}49.57 \\
(232)\end{array}$ & na \\
\hline $\begin{array}{l}\text { Allergen-specific } \\
\text { immunotherapy }\end{array}$ & $\begin{array}{l}7.1 \\
(23)\end{array}$ & na & $0.9(13)$ & na & $0(0)$ & na \\
\hline \multicolumn{7}{|c|}{$\begin{array}{l}{ }^{\dagger} \text { The table only shows the therapeutic groups and type of healthcare products that represent at least } \\
\text { a } 5 \% \text { of the total volume of medications and healthcare products, respectively. The whole table can be } \\
\text { consulted in Supplementary Table } 2 \text {. }\end{array}$} \\
\hline \multicolumn{7}{|l|}{ na: not available } \\
\hline
\end{tabular}

In comparison with the general Catalan population, among beneficiaries, medicines in the 'cardiovascular system', 'genito urinary system and sex hormones' and 'sensory organs' anatomical groups represented a lower proportion of the total number of units of medications dispensed while medications in the 'Antineoplastic and immunomodulating agents' and 'nervous system' groups represented a higher proportion (Table 4). Furthermore, in female beneficiaries, medicines for the 'cardiovascular system' represented a lower proportion of medicines than in the general female population. The same was seen in males with medicines for the 'genitourinary system and sex hormones' group (Table 4).

The largest variation in age groups was observed among children ( $0-14$ years old) (Table 5$)$. Compared with the general population of the same age group, medicines in the groups 'alimentary tract and metabolism', 'cardiovascular system', 'antineoplastic and immunomodulating agents' and 'nervous system' represented a higher proportion (twice or higher) among beneficiaries than in the general population. In contrast, medicines in the 'dermatological', 'systemic hormonal preparations', 'antiinfectives for systemic use', 'musculo-skeletal system' and 'sensory organs' groups represented a lower proportion (half or less) among beneficiaries compared with the general population of children.

\section{Discussion}

Beneficiaries were mainly adults, with functional disability and a low level of education, unemployed, and with one or more children. There is wide evidence regarding the relationships between level of education, as a measure of socioeconomic position, and health [21], and unemployment and health [22]. The proportion of divorced people was higher than in the general population, which could indicate lower social support for PB beneficiaries, compared with the rest of the population $[23,24]$. The beneficiaries were polymedicated and most were using medication for cardiovascular, mental and pain-related disorders, which would also explain the high use of drugs for acid related disorders, which are prescribed to prevent gastroduodenal side effects of polypharmacy and are generally overused [25]. Female 
beneficiaries were more likely to use medicines for pain and mental related disorders than male beneficiaries, which could be related to a higher burden of chronic diseases in women [26].

The 12-month prevalence of use of psychotropic drugs (psycholeptics and psychoanaleptics) was high among PB beneficiaries ( $>42 \%$ ) and higher than that reported in Spanish primary care [27].

Socioeconomic status, debt, workplace conditions and social capital are determinants of mental health [28-30]. This may partly explain the higher use of psychotropic drugs among PB beneficiaries, who were in a situation of economic hardship and more likely to be divorced than the general Catalan population. In addition, mental-health related stigma increases vulnerability to unemployment, especially in males and individuals with low levels of education [31]. Thus, people with mental disorders may have experienced greater economic hardship than those without mental disorders following the economic crisis.

According to their profile of use of medicines and healthcare products, and in contrast to a typical primary care paediatric patient, the paediatric population of beneficiaries presented severe chronic conditions. Thus, this population presents the most complex paediatric cases (such as heart diseases, autoimmune diseases, conduct disorders and attention deficit hyperactivity disorder (ADHD)). The complexity of these chronic and mental diseases may increase the vulnerability of the family, which has to cover medical costs (such as medication and private specialized care) and other costs (home adaptations, informal care and educational accommodations) [32]. In cases of economic hardship, children and adolescents are especially vulnerable [33]. The high prevalence of use of these treatments among young beneficiaries could be explained by the greater prevalence of these disorders among disadvantaged populations [34] but also by the high costs of these treatments. This vulnerability may be even more pronounced when the minor is presenting with severe diseases. It is necessary to evaluate the coverage of care needs for the child, especially when careers are experiencing financial problems. This would be the case, for instance, for the approximately $30 \%$ of children and adolescents using psycholeptics and psychoanaleptics, usually indicated to treat conduct disorders and ADHD in the paediatric population.

More than half of NGO beneficiaries were taking medications for pain-related disorders and over a third were using opioids. Although there has been an increase in the use of opioids in Europe and the USA [35, 36], the prevalence of opioid use among PB beneficiaries is higher than that reported in previous studies. Similarly, the prevalence of use of other antiepileptics, which are also used to deal with pain-related disorders, was also high. Pain is highly disabling [37] and some opioids and antiepileptics, which are used in severe cases, are expensive. People with symptomatic and disabling disorders are more motivated to initiate a treatment [38], which could partially explain why they seek help to cover the treatment expenses when they are experiencing financial hardship. However, chronic pain is associated with high productivity losses due to sick leave and unemployment, partly due to comorbidity with mood disorders [39], which increases the vulnerability of people suffering from pain [40]. Low-wage workers and those with worse working conditions are at higher risk of developing pain-related disorders and experience barriers to accessing quality care, which could hinder recovery $[41,42]$. 
Cardiovascular disease and mental disorders are both chronic disabling conditions with serious clinical and economic consequences $[43,44]$. Non-adherence to medication for cardiovascular disease and mental disorders worsens clinical status and increases the economic burden of these diseases [45-47]. The cost of medications is of potential importance in adherence to medications for these conditions [48] and the reduction of co-payment increases adherence to chronic medications, especially among the more vulnerable populations [17]. A high proportion of the beneficiaries were using medication for the primary and secondary prevention of cardiovascular diseases and diabetes as well as medication for mental disorders. However, the cost of these treatments is low and some patients in need for these treatments may not reach the 20 Euros or more per month threshold set to claim benefit from the SMF. Therefore, there may be a number of patients with no access to these treatments that have not yet been identified.

Economic burden increases the risk of developing several physical and mental disorders, which, in turn, could increase economic hardship, aggravating the situation of the patients experiencing chronic diseases and economic problems. Although the welfare system should ensure access to medicines for patients with economic problems, $30 \%$ of the beneficiaries renewed the aid, which could indicate chronification of pharmaceutical poverty. In the future, studies should be carried out to assess the prevalence of chronification of pharmaceutical poverty and its impact on health.

Strengths and limitations

Interpretation of the results of this study should take the following limitations into account. First, the sample is comprised of people who seek help and fulfil PB criteria to benefit from the SMF. Therefore, the results cannot be extrapolated to all patients in a situation of pharmaceutical poverty, such as those with acute conditions, homeless people and illegal immigrants. Second, the information came from a small geographically restricted area where the SMF was available in 2018. As the aid is extended to other areas, deeper insight will be gained. Third, the study was based on patient registries and information on some key variables, such as comorbidities or social support, were missing.

In spite of these limitations, to the best of our knowledge, this is the first study to describe the pattern of use of medicines in a population affected by pharmaceutical poverty in a Western country. In this paper, we provide some clues about how morbidity patterns differ between the populations in situations of pharmaceutical poverty, which will be useful in better directing healthcare resources to vulnerable populations.

\section{Conclusions}

Compared with the general population in Catalonia, patients presenting with pharmaceutical poverty were older and had a lower level of education, showed a higher prevalence of functional disability, were less likely to be Spanish, and more likely to be divorced and unemployed. These patients commonly consumed treatments for pain-related disorders, gastrointestinal disorders, mental health related disorders, and cardiovascular diseases. 
Compared with male beneficiaries, females were more likely to use medication for pain-related disorders, gastrointestinal disorders and mental health disorders and less likely to use medications for cardiovascular diseases and diabetes. Compared with the Catalan paediatric population, child and adolescent beneficiaries were more likely to use medicines for severe chronic diseases.

Decision makers should take these results into account when evaluating the existing co-payment. A reformulation of the co-payment law would be advisable. In the meantime, physicians and paediatricians should explore economic barriers to access to treatment and direct their patients to resources to help cover the cost of their treatment, especially in the population at higher risk. Future studies should assess the impact of pharmaceutical poverty on health outcomes and explore this problem in populations presenting with acute conditions and in those that do not attend or do not have access to public healthcare centres, such as homeless people and illegal immigrants.

\section{Abbreviations}

PB

Pharmaceutical Bank

WHO

World Health Organization

NHS

National Health System

NGOs

Non-Governmental Organizations

SMF

Social Medicine Fund

ESCA

Catalonian Health Survey (Enquesta de Salut de Catalunyua, in catalan)

ATC

Anatomical Therapeutic Chemical

ADHD

Attention Deficit Hyperactivity Disorder

\section{Declarations}

\section{Ethics approval and consent to participate}

The study was approved by the Ethics Committee at the Sant Joan de Déu hospital (PIC31-20).

\section{Consent for publication}

Not applicable. 
Availability of data and materials

The data used for the elaboration of this paper contains patient information and cannot be made available by the authors.

\section{Competing of interest}

Declaration of interest: none.

\section{Funding}

This research received no external funding.

\section{Authors contribution}

Conceptualization, M.R.-V.; methodology, I.A.-L. and M.R.-V.; formal analysis, M.R.-V and I.A.-L.; interpretation of results, all authors; writing-original draft, M.R.-V.; writing-review and editing, all authors.

\section{Acknowledgments}

We thank the Catalan Health Service and Juan José Ferro, pharmacologist from SAP Deltra Llobregat, for providing data from the general population.

We thank the CIBERESP (Centro de Investigación Biomedica en Res. Epidemiología y Salud Pública, CB16/02/00322) and the European Regional Development Fund for its support in the development of this study. MRV has a "Miguel Servet" research contract from the Instituto de Salud Carlos III (ISCIII) of the Ministry of Economy and Competitiveness (Spain) (CP19/00029). MTPM has an research intensfication from the IDIAPJGol of Institut Català de la Salut (Spain) (7Z20/028).

We thank Stephen Kelly for his help editing the manuscript.

This study was conducted without funding.

\section{References}

1. Thomson S, Cylus J, Evetovits T, Srakar A. Can people afford to pay for health care? new evidence on financial protection in Europe: [regional report]. Copenhagen: World Health Organization, Regional Office for Europe; 2019.

2. Sinnott S-J, Normand C, Byrne S, Woods N, Whelton H. Copayments for prescription medicines on a public health insurance scheme in Ireland. Pharmacoepidemiol Drug Saf. 2016;25:695-704.

3. Terraneo M, Sarti S, Bordogna MT. Social Inequalities and Pharmaceutical Cost Sharing in Italian Regions. Int J Heal Serv. 2014;44:761-85.

4. Aznar-Lou I, Pottegård A, Fernández A, Peñarrubia-María MTMT, Serrano-Blanco A, Sabés-Figuera R, et al. Effect of copayment policies on initial medication non-adherence according to income: a 
population-based study. Saf: BMJ Qual; 2018. ;In press..

5. Kesselheim AS, Huybrechts KF, Choudhry NK, Fulchino LA, Isaman DL, Kowal MK, et al. Prescription drug insurance coverage and patient health outcomes: A systematic review. Am J Public Health American Public Health Association Inc. 2015;105:e17-30.

6. Xu T, Yu X, Ou S, Liu X, Yuan J, Tan X, et al. Adherence to Antihypertensive Medications and Stroke Risk: A Dose-Response Meta-Analysis. J Am Heart Assoc. 2017;6:1-9.

7. Kim YY, Lee JS, Kang HJ, Park SM. Effect of medication adherence on long-term all-cause-mortality and hospitalization for cardiovascular disease in 65,067 newly diagnosed type 2 diabetes patients. Sci Rep Nature Publishing Group. 2018;8:12190.

8. Cutler RL, Fernandez-Llimos F, Frommer M, Benrimoj C, Garcia-Cardenas V. Economic impact of medication non-adherence by disease groups: a systematic review. BMJ Open. 2018;8:16982.

9. Aznar-Lou I, Fernández A, Gil-Girbau M, Sabés-Figuera R, Fajó-Pascual M, Peñarrubia-María MT, et al. Impact of initial medication non-adherence on use of healthcare services and sick leave: a longitudinal study in a large primary care cohort in Spain. Br J Gen Pract. 2017;67:e614-22.

10. González López-Valcárcel B, Librero J, García-Sempere A, Peña LM, Bauer S, Puig-Junoy J, et al. Effect of cost sharing on adherence to evidence-based medications in patients with acute coronary syndrome. Heart. 2017; [In Press].

11. Kazerooni R, Bounthavong M, Watanabe JH. Association of Copayment and Statin Adherence Stratified by Socioeconomic Status. Ann Pharmacother. 2013;47:1463-70.

12. Maciejewski ML, Farley JF, Parker J, Wansink D. Copayment reductions generate greater medication adherence in targeted patients. Health Aff. (Millwood). Project HOPE - The People-to-People Health Foundation, Inc.; 2010;29:2002-8.

13. Chernew ME, Shah MR, Wegh A, Rosenberg SN, Juster IA, Rosen AB, et al. Impact of decreasing copayments on medication adherence within a disease management environment. Health Aff. (Millwood). Project HOPE - The People-to-People Health Foundation, Inc.; 2008;27:103-12.

14. Xu K, Evans DB, Carrin G, Aguilar-Rivera AM. Designing health financing systems to reduce catastrophic health expenditure. Tech. Briefs Policy-makers. World Health Organization; 2005.

15. Bolíbar B, Fina Avilés F, Morros R, Garcia-Gil M, del M, Hermosilla, Ramos E. R, et al. SIDIAP database: electronic clinical records in primary care as a source of information for epidemiologic research. Med clínica Elsevier. 2012;138:617-21.

16. Gobierno de España. Real Decreto-ley 16/2012, de 20 de abril, de medidas urgentes para garantizar la sostenibilidad del Sistema Nacional de Salud y mejorar la calidad y seguridad de sus prestaciones. Boletín Of. del Estado. 2012;31278-312.

17. Aznar-lou I, Pottegård A, Fernández A, Peñarrubia-maría MT, Serrano-blanco A, Sabés-figuera R, et al. Effect of copayment policies on initial medication non-adherence according to income: a populationbased study. BMJ Qual Saf. 2018;27:878-91.

18. Centro de Investigaciones Sociológicas. BARÓMETRO SANITARIO 2018 (Total oleadas). Madrid; 2018. 
19. Leisinger KM. Poverty, Disease, and Medicines in Low-and Middle-Income Countries: The Roles and Responsibilities of Pharmaceutical Corporations. Bus Prof Ethics J John F Kennedy. 2012;31:13585.

20. World Health Organization. Access to medicines: making market forces serve the poor. Geneve; 2017.

21. Furnee CA, Groot W, van den Brink HM. The health effects of education: a meta-analysis. Eur J Public Health. 2008;18:417-21.

22. Tøge AG, Blekesaune M. Unemployment transitions and self-rated health in Europe: A longitudinal analysis of EU-SILC from 2008 to 2011. Soc. Sci. Med. Elsevier Ltd; 2015;143:171-8.

23. Kołodziej-Zaleska A, Przybyła-Basista H. Psychological well-being of individuals after divorce: the role of social support. Curr Issues Personal Psychol Termedia Sp zoo. 2016;4:206-16.

24. Duffy ME. Social Networks and Social Support of Recently Divorced Women. Public Health Nurs. 1993;10:19-24.

25. Forgacs I, Loganayagam A. Overprescribing proton pump inhibitors. BMJ. 2008;336:2-3.

26. Malmusi D, Artazcoz L, Benach J, Borrell C. Perception or real illness? How chronic conditions contribute to gender inequalities in self-rated health. Eur J Public Health. 2012;22:781-6.

27. Rubio-valera M, Fernández A, Luciano JV, Hughes CM, Pinto-meza A, Moreno-küstner B, et al. Psychotropic prescribing in catalonia: Results from an epidemiological study. Fam Pract. 2012;29:154-62.

28. Ahnquist J, Wamala SP, Lindstrom M. Social determinants of health - A question of social or economic capital? Interaction effects of socioeconomic factors on health outcomes. Soc Sci Med. 2012;74:930-9.

29. Sweet E, Nandi A, Adam EK, McDade TW. The high price of debt: Household financial debt and its impact on mental and physical health. Soc Sci Med. 2013;91:94-100.

30. Bambra C, Gibson M, Sowden A, Wright K, Whitehead M, Petticrew M. Tackling the wider social determinants of health and health inequalities: Evidence from systematic reviews. J Epidemiol Community Health. 2010;64:284-91.

31. Evans-Lacko S, Knapp M, McCrone P, Thornicroft G, Mojtabai R. The mental health consequences of the recession: economic hardship and employment of people with mental health problems in 27 European countries. PLoS One. Health Service and Population Research Department, King's College London, Institute of Psychiatry, London, United Kingdom. Sara.Evans-Lacko@kcl.ac.uk; 2013;8:e69792.

32. McCann D, Bull R, Winzenberg T. The daily patterns of time use for parents of children with complex needs. J Child Heal Care. 2012;16:26-52.

33. Rajmil L, de Sanmamed M-J, Choonara I, Faresjö T, Hjern A, Kozyrskyj A, et al. Impact of the 2008 Economic and Financial Crisis on Child Health: A Systematic Review. Int J Environ Res Public Health. 2014;11:6528-46. 
34. Reiss F. Socioeconomic inequalities and mental health problems in children and adolescents: A systematic review. Soc Sci Med. 2013;90:24-31.

35. Kalkman GA, Kramers C, van Dongen RT, van den Brink W, Schellekens A. Trends in use and misuse of opioids in the Netherlands: a retrospective, multi-source database study. Lancet Public Heal Elsevier Ltd. 2019;4:e498-505.

36. Zhu W, Chernew ME, Sherry TB, Maestas N. Initial opioid prescriptions among U.S. Commercially insured patients, 2012-2017. N Engl J Med Massachussetts Medical Society. 2019;380:1043-52.

37. Rice ASC, Smith BH, Blyth FM. Pain and the global burden of disease. Pain. 2016;157:791-6.

38. Gil-Girbau M, Aznar-Lou I, Peñarrubia-María MT, Moreno-Peral P, Fernández A, Bellón J, et al. Reasons for medication non-initiation: A qualitative exploration of the patients' perspective. Res. Social Adm. Pharm. [Internet]. 2019 [cited 2019 Aug 30]; Available from: https://linkinghub.elsevier.com/retrieve/pii/S1551741119300592.

39. Dorner TE, Alexanderson K, Svedberg P, Tinghög P, Ropponen A, Mittendorfer-Rutz E. Synergistic effect between back pain and common mental disorders and the risk of future disability pension: $A$ nationwide study from Sweden. Psychol. Med. Cambridge University Press; 2016;46:425-36.

40. Giladi H, Scott W, Shir Y, Sullivan MJL. Rates and Correlates of Unemployment Across Four Common Chronic Pain Diagnostic Categories. J Occup Rehabil. 2015;25:648-57.

41. Frederiksen P, Karsten MMV, Indahl A, Bendix T. What Challenges Manual Workers' Ability to Cope with Back Pain at Work, and What Influences Their Decision to Call in Sick? J. Occup. Rehabil. Springer New York LLC; 2015;25:707-16.

42. Wami SD, Abere G, Dessie A, Getachew D. Work-related risk factors and the prevalence of low back pain among low wage workers: Results from a cross-sectional study. BMC Public Health BioMed Central Ltd. 2019;19:1072.

43. Roth GA, Johnson C, Abajobir A, Abd-Allah F, Abera SF, Abyu G, et al. Global, Regional, and National Burden of Cardiovascular Diseases for 10 Causes, 1990 to 2015. J Am Coll Cardiol Elsevier USA. 2017;70:1-25.

44. Vigo D, Thornicroft G, Atun R. Estimating the true global burden of mental illness. The Lancet Psychiatry Elsevier Ltd. 2016;3:171-8.

45. Ettehad D, Emdin CA, Kiran A, Anderson SG, Callender T, Emberson J, et al. Blood pressure lowering for prevention of cardiovascular disease and death: A systematic review and meta-analysis. Lancet Elsevier Ltd. 2016;387:957-67.

46. Bitton A, Choudhry NK, Matlin OS, Swanton K, Shrank WH. The Impact of Medication Adherence on Coronary Artery Disease Costs and Outcomes: A Systematic Review. Am J Med. 2013;126:357.e727.

47. Lindström E, Bingefors K. Patient compliance with drug therapy in schizophrenia: Economic and clinical issues. Pharmacoeconomics Adis International Ltd. 2000;18:105-24.

48. Mathes T, Jaschinski T, Pieper D. Adherence influencing factors - a systematic review of systematic reviews. Arch Public Heal. 2014;72:37. 


\section{Supplementary Files}

This is a list of supplementary files associated with this preprint. Click to download.

- SupplementaryTables.docx 